\title{
Comparative Analysis of Income Distributions in the European Union and the United States
}

\author{
M. JAGIElski ${ }^{a, b, *}$, R. DUCZMAL ${ }^{a}$, AND R. KUtneR $^{a}$ \\ ${ }^{a}$ Faculty of Physics, University of Warsaw, Pasteura 5, PL-02093 Warszawa, Poland \\ ${ }^{b}$ Center for Polymer Studies and Department of Physics, Boston University, Boston, Massachusetts 02215, USA
}

We prove that the most rafined approach - our extension of the Yakovenko et al. model - is a universal in the sense that it well describes both household incomes in the European Union and the individual incomes in the United States for all income social classes. This prove was based on our comparative study of various kinds of incomes. The study constitutes a basis for the finding of an impact of the recent world-wide financial crisis on the volatility of various temporary Pareto exponents and on other parameters of the model.

DOI: 10.12693/APhysPolA.127.A-75

PACS: 89.20.-a, 89.65 Gh

\section{Introduction}

One of the major trends in socio- and econophysics having a long tradition is the study of income and wealth redistribution in society and the analysis of social inequalities. Several models, trying to explain the microscopic mechanisms of income dynamics of individuals or households, were proposed [1-29] (and refs. therein). However, none of them give an analytical description of the incomes for all social classes by a single formula based on a unified formalism. Recently [30-33], we extended the Yakovenko et al. model providing, indeed, such a universal formula. Here we show that this formula, containing a low number of free parameters, well reproduces the empirical complementary cumulative distribution functions (CCDFs) both for the European Union (EU) and for the United States (US). Notably, the CCDF is the main statistical tool commonly used in this context. This is the probability that independent stochastic variable takes value larger than some fixed one.

\section{Universal formula}

As for the Yakovenko et al. model, the coexistence of additive and multiplicative stochastic processes is also allowed for our extended Yakovenko et al. model. That is, we assume that household or individual income is determined by: (i)wages and salaries and/or (ii) profits which go to households or individuals mainly through investments and capital gains. Furthermore, for the extended Yakovenko et al. model we assume that the formalism of the income change is the same for entire society but its particular dynamics distinguishes the range of the highincome social class from that of the others (see [32] for details). Finally, the equilibrium probability distribution function we derived in the form [32],

*corresponding author; e-mail: zagielski@interia.pl
$P_{\mathrm{eq}}(m) \propto \begin{cases}\frac{\exp \left(-\left(m_{0} / T\right) \arctan \left(m / m_{0}\right)\right)}{\left[1+\left(m / m_{0}\right)^{2}\right]^{(\alpha+1) / 2},}, & \text { if } m<m_{1}, \\ \frac{\exp \left(-\left(m_{0} / T_{1}\right) \arctan \left(m / m_{0}\right)\right)}{\left[1+\left(m / m_{0}\right)^{2}\right]^{\left(\alpha_{1}+1\right) / 2}}, & \text { if } m \geq m_{1},\end{cases}$

where parameter $T$ can be interpreted as an average income per household or individual within the low- and medium-income social classes, while interpretation of parameter $T_{1}$ is given further in the text. The shape parameter $\alpha$ is the Pareto exponent, describing the income inequality within the medium-income social class, and the shape parameter $\alpha_{1}$ is the Pareto exponent, describing the income inequality within the high-income social class. Parameter $m_{0}$ is a crossover (border) income between the low- and medium-income social classes, and $m_{1}$ is a crossover income between the medium- and high-income social classes. The complementary cumulative distribution function considered below is, indeed, the integrated quantity of above given distribution function.

\section{Agreement with empirical data}

In Fig. 1 (log-log scale) we compare the predictions (shown, for instance, for 2007) based on the extended Yakovenko et al. formula with: (i) empirical complementary cumulative distribution functions of the annual adjusted gross income of individuals in the US and (ii) empirical complementary cumulative distribution functions of the annual total gross income of households in the EU.

Apparently, the extended Yakovenko et al. formula, Eq. (1), describes both the EU and the US empirical CCDFs well. Hence, we were able to provide estimates of the parameters for the years 2005-2010, both for European Union households and United States individuals (cf. Tables I and II). Notably, fits were the best for $T_{1}=m_{1}$, which also gives the interpretation of parameter $T_{1}$.

Remarkably, the values of borders $m_{0}$ and $m_{1}$ are systematically larger for the EU than for the US, except for 2009 (this meaningful exception is discussed further in the text). The systematic deviation is mainly caused 


\section{TABLE II}
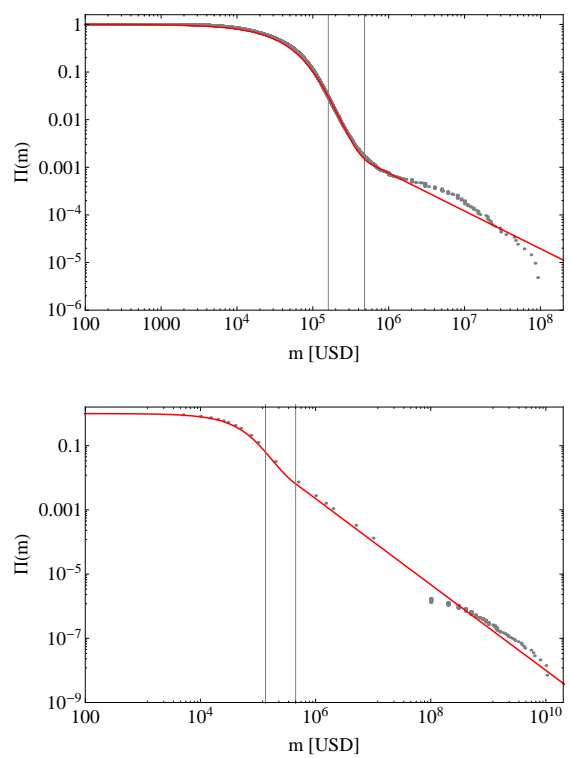

Fig. 1. The comparison for year 2007 of the complementary cumulative distribution functions based on our extended Yakovenko formula (solid lines) with the EU household income empirical data set (dots; top plot) and the US individual income data set (dots; bottom plot). The first and the second vertical lines are placed at $m_{0}$ and $m_{1}$, respectively [34-36].

TABLE I

Parameters $T$ and $T_{1}\left(=m_{1}\right)$ obtained (in US dollars) for the years 2005-2010 from the fit of the CCDF based on our extended Yakovenko et al. formula to the corresponding empirical ones concerning the annual: (i) gross income of individuals in the US and (ii) total gross income of households in the EU. The error bars of the parameters do not exceed $18 \%$.

\begin{tabular}{c|c|c|c|c}
\hline \hline & \multicolumn{2}{|c|}{$\begin{array}{c}\text { European } \\
\text { Union }\end{array}$} & \multicolumn{2}{c}{$\begin{array}{c}\text { United } \\
\text { States }\end{array}$} \\
\hline Year & $T$ & $T_{1}=m_{1}$ & $T$ & $T_{1}=m_{1}$ \\
\hline 2005 & 46278 & 552770 & 45520 & 380000 \\
2006 & 43985 & 529006 & 47220 & 350000 \\
2007 & 48127 & 624350 & 48430 & 450000 \\
2008 & 55257 & 654355 & 48740 & 460000 \\
2009 & 47448 & 371890 & 48050 & 500000 \\
2010 & 51574 & 610749 & 48680 & 420000
\end{tabular}

by the fact that we compare the household incomes ${ }^{\dagger}$ in the EU with individual incomes in the US.

Apparently, the medium-income social class is strongly reduced (almost absent) in the US in comparison with the EU. This result is persistent, i.e. valid for each considered year (in our case from 2005 to 2010). Besides, the border, $m_{0}$, between the low- and medium-income

\footnotetext{
hold.

${ }^{\dagger}$ In average, there are about 1.5 employers per single EU house-
}

Parameters $m_{0}$ (in US dollars), $\alpha$ and $\alpha_{1}$ obtained for the years 2005-2010 from the comparison of the theoretical CCDF based on the extended Yakovenko et al. formula with: (i) empirical CCDFs of the annual total gross income of households in the EU, and (ii) empirical CCDFs of the annual adjusted gross income of individuals in the US. The errors of the parameters do not exceed $4 \%$ in case of $\alpha$ and $\alpha_{1}$, and $17 \%$ in case of $m_{0}$.

\begin{tabular}{c|c|c|c|c|c|c}
\hline \hline & \multicolumn{3}{|c|}{$\begin{array}{c}\text { European } \\
\text { Union }\end{array}$} & \multicolumn{3}{c}{$\begin{array}{c}\text { United } \\
\text { States }\end{array}$} \\
\hline Year & $m_{0}$ & $\alpha$ & $\alpha_{1}$ & $m_{0}$ & $\alpha$ & $\alpha_{1}$ \\
\hline 2005 & 199254 & 2.907 & 0.795 & 135000 & 1.93 & 1.354 \\
2006 & 172373 & 2.892 & 0.86 & 150000 & 1.88 & 1.346 \\
2007 & 208116 & 2.735 & 0.79 & 135000 & 1.83 & 1.336 \\
2008 & 174495 & 2.965 & 0.890 & 135000 & 1.85 & 1.381 \\
2009 & 185945 & 2.974 & 2.608 & 135000 & 1.90 & 1.451 \\
2010 & 183225 & 3.153 & 0.77 & 135000 & 1.86 & 1.395
\end{tabular}

social classes increased at the very beginning of the recent world-wide financial crisis - by about $10 \%$ at 2006 in the US and by about $20 \%$ at 2007 in the EU increasing, thereby, the ranges of the corresponding lowincome social classes. Perhaps, these could be identified as early-warning signals preceding the crisis - however, to say something more definite, it requires a much more systematic study. Furthermore, the drastic decrease of the border, $m_{1}$, between the medium- and high-income social classes - by about $65 \%$ at 2009 in the EU and almost by the same amount at 2006 in the US (see Table I) - increases the relative range of the high-income social class. The former border decrease directly relates to the most striking observation that the high-income social class in the EU vanishes at 2009 as then both shape exponents $\left(\alpha\right.$ and $\left.\alpha_{1}\right)$ are almost equal (up to about $10 \%$ accuracy, see Table II). Again, it is worthy of a much more systematic study.

\section{Conclusions}

In the present paper we demonstrated (to best of our knowledge) the first comparison of incomes in the EU and the US done in such a systematic way. It was possible because we applied the extended Yakovenko et al. formula. We proved, herein, that this formula describes the income of the EU households and the US individuals well. By using the extended Yakovenko et al. formula we showed that both in the EU and in the US we deal with three income social classes, where the medium-income social class has only an intermediate character - one can even say that both in the US and in the exceptional year 2009 in the EU, it has a residual character.

We can conclude that the complementary cumulative distribution function, although being a global (macroeconomic) characteristic, is sufficiently sensitive to the crises and crashes, clearly responding over the extended Yakovenko et al. formula to the income situation in each income social class, at least in the EU and the US. 


\section{Acknowledgments}

M.J. is grateful to the Foundation for Polish Science for financial support.

\section{References}

[1] V. Pareto, Cours d'économie politique, L'Université de Lausanne, 1897.

[2] P. Richmond, S. Hutzler, R. Coelho, P. Repetowicz, A review of empirical studies and models of income distributions in society, in: Econophysics $\&$ Sociophysics: Trends 85 Perspectives, B.K. Chakrabarti, A. Chakraborti, A. Chatterjee, WILEY-VCH, Weinheim 2006, p. 129.

[3] V. M. Yakovenko, J.B. Rosser, Rev. Mod. Phys. 81, 1703 (2009).

[4] A. Banerjee, V.M. Yakovenko, New J. Phys. 12, 075032 (2010).

[5] R. Gibrat, Les inégalités économiques: applications aux inégalités des richesses, à la concentration des entreprises... d'une loi nouvelle, la loi de l'effet proportionnel, Recueil Sirey, Paris 1931.

[6] M. Kalecki, Econometrica 13, 161 (1945).

[7] M. Armatte, Mathématiques et sciences humaines 129, 5 (1995).

[8] J. Sutton, J. Econ. Lit. 35, 40 (1997).

[9] S. Solomon, P. Richmond, Physica A 299, 188 (2001).

[10] P. Richmond, S. Solomon, Int. J. Mod. Phys. C 12 333 (2001).

[11] S. Solomon, P. Richmond, EPJ B 27, 257 (2002).

[12] D.W. Huang, Phys. Rev. E 69, 057103 (2004).

[13] A. Banerjee, V.M. Yakovenko, T. Di Matteo, Physica A 370, 54 (2006).

[14] F. Clementi, T. Di Matteo, M. Gallegati, Physica A 370, 49 (2006).

[15] F. Clementi, T. Di Matteo, M. Gallegati, G. Kaniadakis, Physica A 387, 3201 (2008).

[16] F. Clementi, M. Gallegati, Paretos Law of Income Distribution: Evidence for Germany, the United Kingdom, and the United States, in: Econophysics of Wealth Distributions, A. Chatterjee, B.K. Chakrabarti, S. Yarlagadda, Springer, Italy 2005 , p. 3.

[17] F. Clementi, M. Gallegati, G. Kaniadakis, J. Stat. Mech. Theor. Exp. 2009, P02037 (2009).
[18] A. Drăgulescu, V.M. Yakovenko, EPJ B 17, 723 (2000).

[19] A. Drăgulescu, V.M. Yakovenko, Physica A 299, 213 (2001).

[20] A. Drăgulescu, V.M. Yakovenko, EPJ B 20, 585 (2001).

[21] M. Levy, S. Solomon, Physica A 242, 90 (1997).

[22] P. Łukasiewicz, A. Orłowski, Physica A 344, 146 (2004).

[23] M. Nirei, W. Souma, Rev. Income Wealth 53, 440 (2007).

[24] P.K. Rawlings, D. Reguera, H. Reiss, Physica A 343, 643 (2004).

[25] N. Scafetta, S. Picozzi, B.J. West, Quantitative Finance 4, 353 (2004).

[26] A.C. Silva, V.M. Yakovenko, Europhys. Lett. 69 , 304 (2005).

[27] V.M. Yakovenko, A.C. Silva, Two-class Structure of Income Distribution in the USA: Exponential Bulk and Power-law Tail, in: Econophysics of Wealth Distributions, A. Chatterjee, B.K. Chakrabarti, S. Yarlagadda, Springer, Italy 2005, p. 15.

[28] F. Clementi, M. Gallegati, G. Kaniadakis, J. Stat Mech. Theor. Exp. 2012, P12006 (2012).

[29] A. Orłowski P. Łukasiewicz, K. Karpio, Acta Phys. Pol. A 121, B82 (2012).

[30] M. Jagielski, R. Kutner, M. Pęczkowski, Acta Phys. Pol. A 121, B47 (2012).

[31] M. Jagielski, R. Kutner, Acta Phys. Pol. A 123, 538 (2013).

[32] M. Jagielski, R. Kutner, Physica A 392, 2130 (2013).

[33] M. Jagielski, R. Kutner, Acta Phys. Pol. B Proc. Suppl. 7, 307 (2014).

[34] Statistics on income, social inclusion and living conditions, Eurostat, 2013, EUSILC UDB 2007 — version 3 of March 2010.

[35] The World's Billionaires, Forbes, 2013, http://www.forbes.com/billionaires/.

[36] Internal Revenue Service, 2013, http://www.irs.gov. 